Methods An interventional study was done between October 2018 and February 2019. The intervention included training of 130 housekeeping staff; redefining cleaning and disinfection responsibilities between housekeeping and nursing; adding a checklist for surfaces to be cleaned or disinfected; and emphasizing the inspector's auditing role. The intervention engaged relevant staff partners from infection control, housekeeping, nursing, and environmental services. The study outcome was the frequency of effective cleaning done by housekeepers. It was assessed by comparing the photos taken from specified room sites (pre-prepared by fluorescent gel) using black light before and after cleaning. Six highly touched areas in patient rooms were chosen. The study was divided into three phases: pre-intervention assessment (October 2018), intervention (November 2018 through January 2019), and post-intervention reassessment (February 2019).

Results A total of 27 rooms with 162 opportunities were assessed during the pre-intervention phase. The findings showed that only $39(24.1 \%)$ of the 162 opportunities were effectively cleaned. The frequencies of effective cleaning in different sites were: light switches $11.1 \%$, door knobs $25.9 \%$, water faucets $37 \%$, telephones $25.9 \%$, bed rails $14.8 \%$, and patient tables $29.6 \%$. A total of 33 rooms with 198 opportunities were assessed during the post-intervention phase. The findings showed that 116 (58.6\%) of the 198 opportunities were effectively cleaned. The frequencies of effective cleaning in different areas were: light switches $42.4 \%$, door knobs $84.8 \%$, water faucets $75.7 \%$, telephones $60.6 \%$, bed rails $54.5 \%$, and patient tables $63.6 \%$. The overall improvement in effective cleaning in different sites was $34.5 \% \quad(p<0.001)$, being highest for door knobs $(58.9 \%, \mathrm{p}<0.001)$ and lowest for light switches $(31.3 \%$, $\mathrm{p}=0.014)$.

Conclusion A multidisciplinary intervention including training and auditing of housekeepers was successful in significantly improving cleaning and disinfection at different sites in the patients' rooms. Frequent assessment and feedback may need to be continued until reaching an optimal level. Further studies are needed to evaluate the impact of improved cleaning on infection rates.

\section{MONITORING THE QUALITY INDICATORS OF BLOOD TRANSFUSION SERVICES AS A METHOD TO IMPROVE PATIENT SAFETY AT KING ABDULAZIZ UNIVERSITY HOSPITAL}

Salwa Hindawi, Tarek Elgemmezi, Seraj Alweail. Faculty of Medicine/Hematology and Blood Transfusion King Abdulaziz University - Jeddah - Saudi Arabia

\subsection{6/bmjoq-2019-PSF.51}

Background A quality indicator is measurable information gathered at the critical control points in a process or procedure for monitoring, assessment, and improvement. Quality monitoring is an important tool used to review blood transfusion practice and provide feedback on transfusion trends in blood transfusion services. Quality indicators can improve quality standards and support patient safety through setting priorities and process improvement. The aim of this study was to report 5 years' experience of monitoring the quality indicators at KAUH and to measure its impact on the blood transfusion practice as a tool in hemovigilance system implementation for patient safety.
Methods This was a retrospective study of data collected over the past 5 years (2013-2017) at KAUH, Jeddah, in which the quality indicators for certain parameters were analyzed and benchmarks were set for blood donor adverse reactions, transfusion reactions, fresh frozen plasma (FFP) indate wastage, and cross match to transfusion (CT) ratio. Data were forwarded to the Hospital Transfusion Committee (HTC) for review. Deviations were identified and corrective actions were taken. The outcomes were used to plan for improvement.

Results Among a total of 60,631 blood donors, 282 donor reactions were reported, resulting in a rate of $0.46 \%$, mostly in the form of mild dizziness. 285 adverse transfusion reactions were reported among 99,564 total blood transfusions, resulting in a rate of $0.28 \%$; most were allergic and febrile reactions. Monitoring of the adverse donor reactions showed a decreased incidence; however, the adverse transfusion reactions were under-reported. The FFP in-date wastage was 2205 among 22,590 requested FFP units, resulting in a high rate of 9.76\%. The CT ratio was 1.24. Safety improvements were implemented by a multidisciplinary quality improvement team to determine the critical control points and to address the factors contributing to high FFP wastage.

Conclusion The use of quality indicators as a tool for implementing a hemovigilance system can provide a better understanding of areas for improvement in the quality of the work and safety of patients. Establishing guidelines for appropriate clinical use of blood and proper communication between clinical transfusion staff and practitioners is expected to enhance these features along the blood transfusion chain. The use of a similar model in other institutions will facilitate the local benchmarking between hospitals, which is a feasible method to lower transfusion risk and cost and to improve quality outcomes.

\section{LAB QUALITY IMPROVEMENT PROJECT (MONITORING SEROLOGY REJECTION)}

Sarah Alharbi, Kaneez Zamir, Omar Qassas, Abdulaziz Johani, Abdulrahman Aboud Department of Pathology and Laboratory Medicine, Prince Mohammed Bin Abdul Aziz Hospital - Al Madinah

\subsection{6/bmjoq-2019-PSF.52}

Background This project was initiated because of the observation that serology rejection rates were increasing incrementally. The quality indicator displayed this; therefore, an intervention was necessary to reduce the amount of wastage of samples, patient recalls, supplies, and manpower. The aim of this project was to reduce the number of rejected samples, reduce recalls of patients and redraw of the samples, and to provide the best service to our customers.

Methods

- Using the quality indicators for the statistical measuring of the amount of serology samples rejected.

- PDCA (plan-do-check-act) cycle.

- Quality indicator:

- Numerator is serology rejected tests (lithium heparin and SST);

- Denominator is serology total tests done (lithium heparin and SST);

- International benchmark value $0.56 \%$;

- Baseline quality indicator was measured and then monitoring was done after implementation of interventions. 